\title{
Four New Sesquiterpenoids from Cultures of the Fungus Phellinidium sulphurascens
}

\author{
Zhen-Zhu Zhao $\cdot$ He-Ping Chen $\cdot$ Tao Feng $\cdot$ \\ Zheng-Hui Li $\cdot$ Ze-Jun Dong $\cdot$ Ji-Kai Liu
}

Received: 24 October 2014/ Accepted: 18 November 2014/Published online: 10 December 2014

(C) The Author(s) 2014. This article is published with open access at Springerlink.com

\begin{abstract}
Four new sesquiterpenoids, namely 12-hydroxy-3-oxodrimenol (1), 11-hydroxyacetoxydrim-7-en-3 $\beta$-ol (2), 2,6-dimethyl-7,10-epoxy-10-hydroxymethyldodeca-2,11-dien-6-ol (3), and 7,10-epoxy-2,6,10-trimethyldodeca-2,11diene-4,6-diol (4), along with fourteen known compounds, were isolated from the cultures of Phellinidium sulphurascens. The structures of compounds 1-4 were established on the basis of extensive spectroscopic analysis. All of them were evaluated for their cytotoxic activities.
\end{abstract}

Keywords Phellinidium sulphurascens · Drimane · 7,10-Epoxy-2,6,10-trimethyldodeca-2,11-diene

\section{Introduction}

The wood-decaying fungus Phellinidium sulphurascen is a rare species belonging to the genus Phellinidium which is close-related to the genus Phellinus taxonomically. So far, only seven species of this genus were discovered in China, including $P$. sulphurascens [1]. In addition, the genus Phellinus has plenty of bioactive secondary metabolites involved in different types, such as sesquiterpenoids, steroids, pigments and polysaccharides [2-5]. Most of these metabolites showed significant vascular-relaxing [2], antiviral [3], estrogenic and anti-estrogenic [4], and antitumor

Electronic supplementary material The online version of this article (doi:10.1007/s13659-014-0047-x) contains supplementary material, which is available to authorized users.

Z.-Z. Zhao - H.-P. Chen - T. Feng · Z.-H. Li · Z.-J. Dong ·

J.-K. Liu $(\bowtie)$

State Key Laboratory of Phytochemistry and Plant Resources in West China, Kunming Institute of Botany, Chinese Academy of Sciences, Kunming 650201, People's Republic of China e-mail: jkliu@mail.kib.ac.cn

Z.-Z. Zhao · H.-P. Chen

University of Chinese Academy of Sciences, Beijing 100049,

People's Republic of China activities [5]. However, the fungus $P$. sulphurascens has not been chemically investigated until now. As our continuous search for bioactive natural products from higher fungi, it is of importance to carry out the chemical investigation on cultures of $P$. sulphurascens, which resulted in the isolation of four new sesquiterpennoids (Fig. 1), namely 12-hydroxy3-oxodrimenol (1), 11-hydroxyacetoxydrim-7-en-3 $\beta$-ol (2), 2,6-dimethyl-7,10-epoxy-10-hydroxymethyldodeca-2,11dien-6-ol (3), and 7,10-epoxy-2,6,10-trimethyldodeca2,11-diene-4,6-diol (4), together with fourteen known compounds, from the EtOAc extract of the cultures of this fungus. All compounds were evaluated for their cytotoxicities against five human cancer cell lines.

\section{Results and Discussion}

Compound 1, obtained as amorphous powder, possessed a molecular formula $\mathrm{C}_{15} \mathrm{H}_{24} \mathrm{O}_{3}$, as deduced from HREIMS at $m / z, 252.1723[\mathrm{M}]^{+}$(calcd for $\mathrm{C}_{15} \mathrm{H}_{24} \mathrm{O}_{3}, 252.1725$ ). The IR spectrum showed absorption bands at $3440 \mathrm{~cm}^{-1}$ for hydroxy and $1701 \mathrm{~cm}^{-1}$ for carbonyl functional groups. The ${ }^{1} \mathrm{H}$ NMR spectrum of compound 1 showed three methyl singlets $\left(\delta_{\mathrm{H}} 1.02,1.04\right.$ and 1.08), two $O$-bearing methylene signals $\left(\delta_{\mathrm{H}} 3.70\right.$, ddd, $J=11.1,5.0,2.5 \mathrm{~Hz} ; \delta_{\mathrm{H}}$ 
Fig. 1 Structures of compounds 1-4, 1a, and 2a

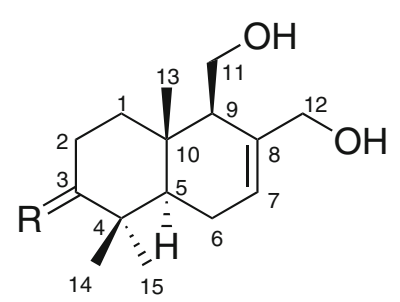

$1 \mathrm{R}=\mathrm{O}$ 1a $\mathrm{R}=\beta-\mathrm{OH}, \alpha-\mathrm{H}$

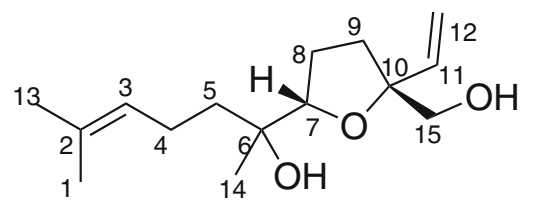

3<smiles>[R]PC(=O)OC[C@H]1C(C)=CC[C@H]2C(C)(C)[C@H](O)CC[C@]12C</smiles>

$$
2 \mathrm{R}=\mathrm{OH}
$$$$
2 a \mathrm{R}=\mathrm{H}
$$<smiles>C=C[C@]1(C)CC[C@@H](C(C)(O)CC(O)C=C(C)C)O1</smiles>

4
3.90 , ddd, $J=11.1,6.9,5.0 \mathrm{~Hz}$ and $\delta_{\mathrm{H}} 3.96$, dd, $J=12.2$, $6.9 \mathrm{~Hz} ; \delta_{\mathrm{H}} 4.27, \mathrm{dd}, J=12.2,5.3 \mathrm{~Hz}$ ), and one olefinic proton $\left(\delta_{\mathrm{H}} 5.78, \mathrm{~d}, J=5.1 \mathrm{~Hz}\right)$. The ${ }^{13} \mathrm{C}$ NMR spectrum of 1 showed fifteen carbon resonances, which were ascribed to a trisubstituted double bond, three methyl, five methylenes (two oxygenated), two $s p^{3}$ methines, two $s p^{3}$ quaternary carbons and one carboxyl $\left(\delta_{\mathrm{C}} 215.1\right)$ (Table 1$)$. The chemical shift values of 1D NMR of $\mathbf{1}$ were quite similar to those of the known compound 3 $\beta, 12$-dihydroxydrimenol (1a) [6], which suggested compound 1 possessed a drimane sesquiterpenoid skeleton. The notable difference between $\mathbf{1 a}$ and $\mathbf{1}$ was that the hydroxy group at C-3 $\left(\delta_{\mathrm{C}} 79.7\right)$ in 1a was oxidized into a carbonyl group $\left(\delta_{\mathrm{C}} 215.1\right)$ in $\mathbf{1}$, which caused the downfield shifts of C-4 from $\delta_{\mathrm{C}} 39.8$ in 1a to 47.9 in $\mathbf{1}$, and C-2 from $\delta 28.1$ in $\mathbf{1 a}$ to 38.7 in $\mathbf{1}$. The above assignment was further supported by the HMBC correlations from $\delta_{\mathrm{H}} 1.08(\mathrm{Me}-14, \mathrm{~s})$ and $1.02(\mathrm{Me}-15, \mathrm{~s})$ to $\delta_{\mathrm{C}}$ 215.1 (s, C-3) (Fig. 2), as well as IR absorption band at $1701 \mathrm{~cm}^{-1}$ and mass data analysis. In the ROESY spectrum (Fig. 2), cross peaks between Me-15/H-5, H-5/H-9, Me-13/H-11 were observed, which suggested that both Me13 and $\mathrm{H}-11$ should be $\beta$ oriented. Therefore, compound 1 was established as 12-hydroxy-3-oxodrimenol (1).

Compound 2 was isolated as white powder. The molecular formula was established to be $\mathrm{C}_{17} \mathrm{H}_{28} \mathrm{O}_{4}$ on the basis of HREIMS at $\mathrm{m} / z 296.1974$ [M] $^{+}$(calcd for 296.1988, $\mathrm{C}_{17} \mathrm{H}_{28} \mathrm{O}_{4}$ ). The IR spectrum showed absorptions at $3442 \mathrm{~cm}^{-1}$ for hydroxy, $1725 \mathrm{~cm}^{-1}$ for carbonyl, and $1631 \mathrm{~cm}^{-1}$ for double bond functional groups. The ${ }^{1} \mathrm{H}$ NMR (Table 1) spectrum showed resonances for four methyl singlets $\left(\delta_{\mathrm{H}} 0.81,0.87,0.98,1.65\right)$, two $O$-bearing methylene signals $\left(\delta_{\mathrm{H}} 4.25\right.$, dd, $J=11.5,6.3 \mathrm{~Hz} ; \delta_{\mathrm{H}} 4.39$, $\mathrm{dd}, J=11.5,3.3 \mathrm{~Hz}$ and $\delta_{\mathrm{H}} 4.13, \mathrm{dd}, J=12.7,5.4 \mathrm{~Hz} ; \delta_{\mathrm{H}}$
4.14, dd, $J=12.7,5.4 \mathrm{~Hz}$ ), one $O$-bearing methane signal $\left(\delta_{\mathrm{H}} 3.26, \mathrm{dd}, J=11.3,3.2 \mathrm{~Hz}\right)$, and one olefinic proton $\left(\delta_{\mathrm{H}}\right.$ $5.52 \mathrm{~m}$ ). The ${ }^{13} \mathrm{C}$ NMR spectrum, along with the DEPT and HSQC spectra, classified the functionalities as a trisubstituted double bond, four methyl, five $s p^{3}$ methylenes (two oxygenated), three $s p^{3}$ methines (one oxygenated), two $s p^{3}$ quaternary carbons, and one carbonyl $\left(\delta_{\mathrm{C}} 173.5\right)$ (Table 1). These NMR spectroscopic data of 2 resembled those of a coexisting known compound acetoxydrim-7-en$3 \beta$-ol (2a) [7], except for the chemical shift of C-17 was $60.9\left(\mathrm{CH}_{2}\right)$ in 2 instead of $21.3 \mathrm{ppm}\left(\mathrm{CH}_{3}\right)$ in $\mathbf{2 a}$. Therefore, compound 2 was established as a hydroxy derivative of $\mathbf{2 a}$, which was also supported by the HMBC correlations from $\delta_{\mathrm{H}} 4.13(1 \mathrm{H}, \mathrm{d}, J=5.1 \mathrm{~Hz})$ and $4.14(1 \mathrm{H}, \mathrm{d}$, $J=5.1 \mathrm{~Hz})$ to $\delta_{\mathrm{C}} 173.5(\mathrm{C}-16$, s) (Fig. 2), as well as mass data analysis. In the ROESY spectrum (Fig. 2), the cross peak of $\mathrm{H}-5 / \mathrm{Me}-15$ suggested that $\mathrm{C}-14$ was $\beta$ oriented, while the cross peak of Me- $15 / \mathrm{H}-3$, as well as the constant coupling of $\mathrm{H}-3\left(\delta_{\mathrm{H}} 3.26, \mathrm{dd}, J=11.3,3.2 \mathrm{~Hz}\right)$, indicated $3-\mathrm{OH}$ to be $\beta$ oriented. Besides, the cross peaks of Me-14/ $\mathrm{H}-2, \mathrm{H}-2 / \mathrm{Me}-13$, Me-13/H-11 proved that $\mathrm{H}-9$ was $\alpha$ oriented and Me-13 was $\beta$ oriented. Therefore, compound 2 was elucidated as 11-hydroxyacetoxydrim-7-en-3 $\beta$-ol.

Compound 3, a colorless oil, had an $[\mathrm{M}]^{+}$peak at $\mathrm{m} / \mathrm{z}$ 254.1873 in the HREIMS (cacld for 254.1882), corresponding to a molecular formula $\mathrm{C}_{15} \mathrm{H}_{26} \mathrm{O}_{3}$. 1D NMR spectra in combination with HSQC spectrum demonstrated 15 carbons, which were classified into three methyl, six methylenes (one olefinic carbon, one oxygenated carbon), three methines (two olefinic carbons) and three quaternary carbons (one olefinic carbon) (Table 2). All these data suggested that compound $\mathbf{3}$ was a sesquiterpenoid possessing the same skeleton with 2,6,10-trimethyl-7,10- 
Table $1{ }^{1} \mathrm{H}$ NMR $(600 \mathrm{MHz})$ and ${ }^{13} \mathrm{C}$ NMR $(150 \mathrm{MHz})$ data of $\mathbf{1}$ and $\mathbf{2}(\delta$ in ppm, $J$ in $\mathrm{Hz})$

\begin{tabular}{|c|c|c|c|c|c|c|}
\hline \multirow[t]{2}{*}{ No. } & \multicolumn{2}{|l|}{1} & \multirow{2}{*}{$\begin{array}{l}\mathbf{1 a}^{\mathrm{a}} \\
\delta_{\mathrm{C}}^{\mathrm{d}}\end{array}$} & \multicolumn{2}{|l|}{2} & \multirow{2}{*}{$\begin{array}{l}\mathbf{2 a}^{\mathrm{b}} \\
\delta_{\mathrm{H}}^{\mathrm{d}}\end{array}$} \\
\hline & $\delta_{\mathrm{H}}^{\mathrm{c}}$ & $\delta_{\mathrm{C}}^{\mathrm{c}}$ & & $\delta_{\mathrm{H}}^{\mathrm{d}}$ & $\delta_{\mathrm{C}}^{\mathrm{d}}$ & \\
\hline \multirow[t]{2}{*}{1} & 2.76, ddd $(14.5,14.5,5.3)$ & $35.1, \mathrm{t}$ & 38.8 & 1.62, overlapped & $27.4, \mathrm{t}$ & 27.3 \\
\hline & 2.14, ddd $(14.5,3.7,3.7)$ & & & 1.67, overlapped & & \\
\hline \multirow[t]{2}{*}{2} & 2.34, ddd $(13.3,5.3,3.7)$ & $38.7, \mathrm{t}$ & 28.1 & 1.99 , overlapped & $37.7, \mathrm{t}$ & 37.6 \\
\hline & 1.59, ddd $(14.5,13.3,3.7)$ & & & $1.28, \mathrm{~m}$ & & \\
\hline 3 & & $215.1, \mathrm{~s}$ & 79.5 & 3.26 , dd $(11.3,3.2)$ & $78.9, \mathrm{~d}$ & 78.9 \\
\hline 4 & & $47.9, \mathrm{~s}$ & 39.8 & & $38.8, \mathrm{~s}$ & 38.7 \\
\hline 5 & $1.63, \mathrm{dd}(11.8,4.6)$ & $51.8, \mathrm{~d}$ & 50.7 & $1.22, \mathrm{dd}(11.8,4.8)$ & $49.4, \mathrm{~d}$ & 49.3 \\
\hline \multirow[t]{2}{*}{6} & 2.14 , overlapped & $24.4, \mathrm{t}$ & 24.3 & $1.96, \mathrm{~m}$ & $23.3, \mathrm{t}$ & 23.2 \\
\hline & $2.01, \mathrm{~m}$ & & & 2.01, overlapped & & \\
\hline 7 & $5.78, \mathrm{~d}(5.1)$ & $125.0, \mathrm{~d}$ & 126.4 & $5.52, \mathrm{~m}$ & 124.1, d & 123.5 \\
\hline 8 & & $139.3, \mathrm{~s}$ & 138.4 & & $131.8, \mathrm{~s}$ & 132.3 \\
\hline 9 & 2.14 , overlapped & $54.7, \mathrm{~d}$ & 55.8 & $2.05, \mathrm{~m}$ & $53.3, \mathrm{~d}$ & 53.2 \\
\hline 10 & & $36.2, \mathrm{~s}$ & 36.6 & & $35.9, \mathrm{~s}$ & 35.7 \\
\hline \multirow[t]{2}{*}{11} & 3.70, ddd $(11.1,5.0,2.5)$ & $60.9, \mathrm{t}$ & 61.2 & $4.25, \mathrm{dd}(11.5,6.3)$ & $64.3, \mathrm{t}$ & 63.0 \\
\hline & 3.90, ddd $(11.1,6.9,5.0)$ & & & $4.39, \mathrm{dd}(11.5,3.3)$ & & \\
\hline \multirow[t]{2}{*}{12} & 3.96 , dd $(12.2,6.9)$ & $66.7, \mathrm{t}$ & 67.0 & $1.65, \mathrm{~s}$ & $21.7, \mathrm{q}$ & 21.5 \\
\hline & $4.27, \mathrm{dd}(12.2,5.3)$ & & & & & \\
\hline 13 & $1.04, \mathrm{~s}$ & $14.4, \mathrm{q}$ & 15.0 & $0.81, \mathrm{~s}$ & $14.7, \mathrm{q}$ & 14.5 \\
\hline 14 & $1.08, \mathrm{~s}$ & $22.5, \mathrm{q}$ & 15.9 & $0.87, \mathrm{~s}$ & $15.4, \mathrm{q}$ & 15.2 \\
\hline 15 & $1.02, \mathrm{~s}$ & 25.7, q & 28.7 & $0.98, \mathrm{~s}$ & $28.2, \mathrm{q}$ & 28.0 \\
\hline 16 & & & & & $173.5, \mathrm{~s}$ & 171.2 \\
\hline \multirow[t]{2}{*}{17} & & & & $4.13, \mathrm{dd}(12.7,5.4)$ & $60.9, \mathrm{t}$ & 21.3 \\
\hline & & & & $4.14, \mathrm{dd}(12.7,5.4)$ & & \\
\hline $11-\mathrm{OH}$ & $4.34, \mathrm{dd}(5.0,5.0)$ & & & & & \\
\hline $12-\mathrm{OH}$ & $4.20, \mathrm{dd}(6.9,5.3)$ & & & & & \\
\hline $17-\mathrm{OH}$ & & & & $2.36, \mathrm{dd}(5.4,5.4)$ & & \\
\hline
\end{tabular}

${ }^{\text {a }}$ Literature data

${ }^{\mathrm{b}}$ Experimental data

${ }^{c}$ Spectra were measured in acetone- $d_{6}$

d Spectra were measured in $\mathrm{CDCl}_{3}$

epoxy-2,11-dodecadien-6-ol [8]. The only difference between them was that one proton of Me-15 $\left(\delta_{\mathrm{C}} 24.2, \mathrm{q}\right)$ in 2,6,10-trimethyl-7,10-epoxy-2,11-dodecadien-6-ol was replaced by a hydroxyl $\left(\delta_{\mathrm{C}} 67.4, \mathrm{t}\right)$ in 3 . This change was supported by the HMBC correlations of H-15 $\left(\delta_{\mathrm{H}} 3.47\right.$, d, $\left.J=11.4,4.4 \mathrm{~Hz}, \delta_{\mathrm{H}} 3.42, \mathrm{dd}, J=11.4,6.2 \mathrm{~Hz}\right) / \mathrm{C}-10\left(\delta_{\mathrm{C}}\right.$ 85.8, s) and H-15/C-11 ( $\left.\delta_{\mathrm{C}} 141.1, \mathrm{~d}\right)$ (Fig. 2). In the ROESY spectrum (Fig. 2), correlations of H-15/H-9 $\beta\left(\delta_{\mathrm{H}}\right.$ 1.97) and $\mathrm{H}-9 \beta / \mathrm{H}-7$ indicated that $\mathrm{H}-15$ and $\mathrm{H}-7$ were in the same side. Therefore, compound $\mathbf{3}$ was identified as 2,6-dimethyl-7,10-epoxy-10-hydroxymethyldodeca2,11-dien-6-ol.

Compound 4 was isolated as a colorless oil. Its molecular formula was determined as $\mathrm{C}_{15} \mathrm{H}_{26} \mathrm{O}_{3}$ based on the HRESIMS at $m / z 277.1772$ (cacld for 277.1780, $\left.[\mathrm{M}+\mathrm{Na}]^{+}\right)$. Its NMR data were similar to those of 2,6,10- trimethyl-7,10-epoxy-2,11-dodecadien-6-ol [8]. Compared with compound $\mathbf{3}$, one proton at $\mathrm{C}-4$ of compound $\mathbf{4}$ was substituted by a hydroxy, while Me-15 of 4 was not oxygenated. This change was supported by ${ }^{1} \mathrm{H}-{ }^{1} \mathrm{H}$ COSY correlation of $\mathrm{H}-4 / \mathrm{H}-5$ and HMBC correlation of $\mathrm{H}-4 / \mathrm{C}-2$ (Fig. 2). The ROESY data suggested the other parts of 4 were same to those of $\mathbf{3}$ (Fig. 2). Therefore, the structure of compound 4 was elucidated as 7,10-epoxy-2,6,10-trimethyldodeca-2,11-diene-4,6-diol.

Fourteen known compounds were identified as 3,11-dihydrodrimane [9], 3 $\beta, 11,12$-trihydroxy-drimene (1a), $3 \beta$ hydroxydrimenol, 11,12-dihydroxydrimene [6], acetoxydrim-7-en-3 $\beta$-ol (2a) [7], 3-keto-drimenol [10], drimane2,11-diol [11], 11-hydroxydrim-8-en-7-one [12], (-)drimenol [13], 15-hydroxy-T-muurolol [14], 2 $\beta$-hydroxy$\delta$-cadinol [15], $2 \beta$-hydroxy- $\alpha$-cadinol [16], $3 \beta$-hydroxy- $\delta$ - 
Fig. 2 Key 2D NMR correlations of 1-4

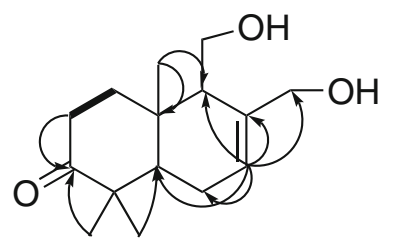

1

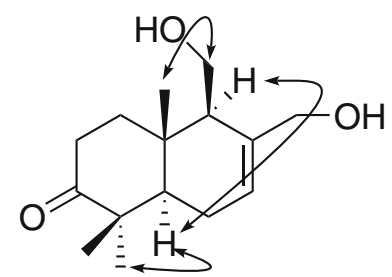

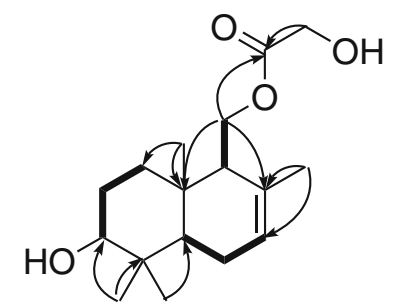

2

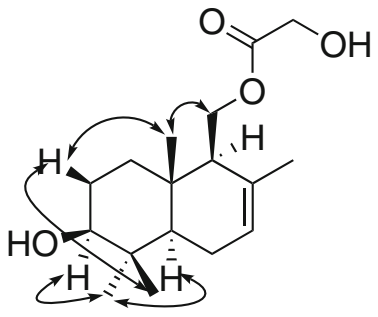

3

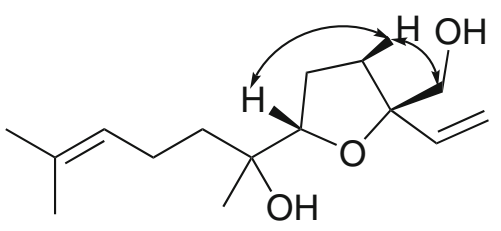

4<smiles>C=CC12CCCCCCCCCCC1O2</smiles>

$\mathrm{HMBC}$ cadinol [17], and 2-(5'-ethenyltetrahydro-5'-methylfuran2'-yl)-6-methylhept-5-en-ol [18] by comparison of spectroscopic data with those reported in the literature.

Unfortunately, none of them showed significant inhibitory activities against the five human cancer cell lines (> $40 \mu \mathrm{mol})$.

\section{Experimental}

\subsection{General Experimental and Procedures}

Optical rotations were obtained on a JASCO P-1020 digital polarimeter. IR and UV spectra were recorded on a Bruker Tensor 27 FT-IR spectrometer with $\mathrm{KBr}$ pellets and a Shimadzu UV-2401PC instrument, respectively. 1D and 2D NMR spectra were obtained on a Bruker Avance III $600 \mathrm{MHz}$ spectrometer. ESIMS and HREIMS were measured on a Waters Xevo TQ-S spectrometer and a Waters Autospec Premier P776 spectrometer, respectively. HRESIMS was measured on an Agilent G6230 TOF MS spectrometer. Silica gel 200-300 mesh (Qingdao Marine Chemical Inc., China) and Sephadex LH-20 (Amersham Biosciences, Sweden) were used for column chromatography. Medium pressure liquid chromatography (MPLC) was performed on a Büchi Sepacore System equipping pump manager C-615, pump modules C-605 and fraction collector C-660 (Büchi Labortechnik AG, Switzerland), and columns packed with Chromatorex C-18 (40-75 $\mu \mathrm{m}$, Fuji Silysia Chemical Ltd., Japan). Preparative high performance liquid chromatography (Prep-HPLC) was performed on an Agilent 1260 liquid chromatography system equipped with a Zorbax SB-C18 column (5 $\mu \mathrm{m}$, $9.4 \times 150 \mathrm{~mm})$.

\subsection{Fungus Material}

The fungus Phellinidium sulphurascens was collected from Changbai Mountain, Jilin Province, China in 2009, and were identified by Prof. Yu-Cheng Dai (Institute of Microbiology, Beijing Forestry University). The culture medium to ferment this fungus consist of glucose $(5 \%)$, 
peptone from porcine meat $(0.15 \%)$, yeast powder $(0.5 \%), \mathrm{KH}_{2} \mathrm{PO}_{4}(0.05 \%)$ and $\mathrm{MgSO}_{4}(0.05 \%)$. Five hundred 500-mL Erlenmeyer flasks each containing $350 \mathrm{~mL}$ of above-mentioned culture medium were inoculated with $P$. sulphurascens strains, respectively. Then they were incubated on rotary shakers at $24{ }^{\circ} \mathrm{C}$ and $150 \mathrm{rpm}$ for 25 days in dark environment.

\subsection{Extraction and Isolation}

The culture broth $(20 \mathrm{~L})$ of $P$. sulphurascens was filtered, the filtrate was extracted four times with ethyl acetate (EtOAc). Meanwhile, the mycelium was extracted by $\mathrm{CHCl}_{3} / \mathrm{MeOH}$ (1:1) for three times. The EtOAc layer together with the mycelium extraction was concentrated under reduced pressure to afford a crude extract $(6.0 \mathrm{~g})$. Then this residue was decolorized by Sephadex LH-20 column chromatography $\left(\mathrm{CHCl}_{3}: \mathrm{MeOH}=1: 1\right)$, followed by MPLC eluting with $\mathrm{MeOH} / \mathrm{H}_{2} \mathrm{O}$ (from 20:80 to 100:0) to give seven main fractions $(\mathrm{A}-\mathrm{G})$. Fraction $\mathrm{C}(0.8 \mathrm{~g})$ was separated by Sephadex LH-20 column chromatography ( $\mathrm{MeOH})$ to afford three subfractions (C1-C3). Subfraction C2 was subjected to Sephadex LH-20 column chromatography (acetone) to afford six fractions $(\mathrm{C} 2 \mathrm{a}-\mathrm{C} 2 \mathrm{c})$. Fraction $\mathrm{C} 2 \mathrm{c}$ was separated on a Prep-HPLC to afford $\mathbf{1}(1.2 \mathrm{mg}), \mathbf{1 a}(3.8 \mathrm{mg}), 3 \beta$-hydroxydrimenol $(5.3 \mathrm{mg})$. A similar purification procedure on fraction D (1.1 g) yielded $2(1.5 \mathrm{mg}), \mathbf{2 a}(2.8 \mathrm{mg}), 11,12-$ dihydroxydrimene $(5.8 \mathrm{mg}), 3$-keto-drimenol $(3.3 \mathrm{mg})$, while fraction E yielded $\mathbf{3}(0.8 \mathrm{mg}), \mathbf{4}(0.9 \mathrm{mg})$, drimene2,11-diol (7.2 mg), 11-hydroxydrim-8-en-7-one $(6.8 \mathrm{mg}$ ), 3,11-dihydrodrimane $(4.0 \mathrm{mg}),(-)$-drimenol $(4.5 \mathrm{mg})$, 15 -hydroxy-T-muurolol $(3.2 \mathrm{mg}) .2 \beta$-hydroxy- $\delta$-cadinol (4.5 mg), $2 \beta$-hydroxy- $\alpha$-cadinol (4.4 mg), $3 \beta$-hydroxy- $\delta$ cadinol $(4.3 \mathrm{mg})$, and $2-\left(5^{\prime}\right.$-ethenyltetrahydro- $5^{\prime}$-methylfuran-2'-yl)-6-methylhept-5-en-ol (7.9 mg).

\subsubsection{2-Hydroxy-3-oxodrimenol (1)}

Amorphous powder, $[\alpha]_{\mathrm{D}}^{18}-56.9(c=0.03, \mathrm{MeOH})$. IR (KBr) $v_{\max } \mathrm{cm}^{-1}: 3440,2971,2933,1701,1630,157$, 1426, 1384, 1033. For ${ }^{1} \mathrm{H}$ NMR (600 MHz, acetone- $d_{6}$ ) and ${ }^{13} \mathrm{C}$ NMR $\left(150 \mathrm{MHz}\right.$, acetone- $\left.d_{6}\right)$ spectroscopic data, see Table 1. HREIMS $m / z: 252.1723\left[^{[M]^{+}}\right.$(calcd for $\left.\mathrm{C}_{15} \mathrm{H}_{24} \mathrm{O}_{3}, 252.1725\right)$.

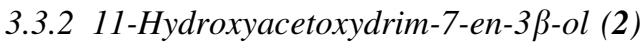

Amorphous powder, $[\alpha]_{\mathrm{D}}^{18}+6.2(c 0.03, \mathrm{MeOH})$. IR $(\mathrm{KBr})$ $v_{\max } \mathrm{cm}^{-1}: 3442,2958,2923,2854,1725,1631,1448$, $1428,1383,1104,1034 .{ }^{1} \mathrm{H}$ NMR $\left(600 \mathrm{MHz}, \mathrm{CDCl}_{3}\right)$ and ${ }^{13} \mathrm{C}$ NMR $\left(150 \mathrm{MHz}, \mathrm{CDCl}_{3}\right)$ spectroscopic data, see Table 1. HREIMS m/z: 296.1974 [M] $^{+}$(calcd for $\left.\mathrm{C}_{17} \mathrm{H}_{28} \mathrm{O}_{4}, 296.1988\right)$.
Table $2{ }^{1} \mathrm{H}$ NMR $(600 \mathrm{MHz})$ and ${ }^{13} \mathrm{C}$ NMR $(150 \mathrm{MHz})$ data of 3 and 4 in $\mathrm{CDCl}_{3}(\delta$ in ppm, $J$ in $\mathrm{Hz})$

\begin{tabular}{|c|c|c|c|c|}
\hline \multirow[t]{2}{*}{ No. } & \multicolumn{2}{|l|}{3} & \multicolumn{2}{|l|}{4} \\
\hline & $\delta_{\mathrm{C}}$ & $\delta_{\mathrm{H}}$ & $\delta_{\mathrm{C}}$ & $\delta_{\mathrm{H}}$ \\
\hline 1 & $17.8, \mathrm{q}$ & $1.62, \mathrm{~s}$ & $18.4, \mathrm{q}$ & $1.68, \mathrm{~s}$ \\
\hline 2 & $131.9, \mathrm{~s}$ & & 136.6, s & \\
\hline 3 & 124.5, d & 5.04 , br. t (7.3) & $125.9, \mathrm{~d}$ & 5.11 , br. d (8.4) \\
\hline 4 & $22.3, \mathrm{t}$ & $\begin{array}{l}2.10, \mathrm{~m} \\
2.03, \text { overlapped }\end{array}$ & $73.7, \mathrm{~d}$ & $\begin{array}{l}\text { 4.77, ddd (10.6, } \\
\quad 8.4,6.4)\end{array}$ \\
\hline \multirow[t]{2}{*}{5} & $37.5, \mathrm{t}$ & $\begin{array}{l}1.51, \mathrm{ddd} \\
(13.7,11.6,5.2)\end{array}$ & $47.8, \mathrm{t}$ & $\begin{array}{l}2.06, \text { dd }(13.1, \\
6.4)\end{array}$ \\
\hline & & $\begin{array}{l}1.35, \text { ddd } \\
(13.7,11.6,5.4)\end{array}$ & & $\begin{array}{l}\text { 1.61, } \\
\text { overlapped }\end{array}$ \\
\hline 6 & $72.9, \mathrm{~s}$ & & $73.1, \mathrm{~s}$ & \\
\hline 7 & $86.9, \mathrm{~d}$ & $3.81, \mathrm{dd}(9.0,6.0)$ & $87.9, \mathrm{~d}$ & $\begin{array}{l}3.59 \text {, dd }(10.3, \\
3.2)\end{array}$ \\
\hline \multirow[t]{2}{*}{8} & $25.9, \mathrm{t}$ & 1.81 , overlapped & $26.5, \mathrm{t}$ & $\begin{array}{l}1.59, \\
\text { overlapped }\end{array}$ \\
\hline & & $1.78, \mathrm{~m}$ & & $1.33, \mathrm{~m}$ \\
\hline \multirow[t]{2}{*}{9} & $32.6, \mathrm{t}$ & 1.97, overlapped & $39.1, \mathrm{t}$ & $\begin{array}{l}\text { 1.81, ddd (14.9, } \\
11.1,5.6)\end{array}$ \\
\hline & & 1.83 , overlapped & & $\begin{array}{l}1.62, \\
\text { overlapped }\end{array}$ \\
\hline 10 & $85.8, \mathrm{~s}$ & & $80.3, \mathrm{~s}$ & \\
\hline 11 & 141.1, d & $\begin{array}{l}\text { 5.83, dd (17.5, } \\
10.9)\end{array}$ & $145.2, \mathrm{~d}$ & $\begin{array}{l}5.90, \text { dd }(17.5, \\
10.9)\end{array}$ \\
\hline \multirow[t]{2}{*}{12} & $115.0, \mathrm{t}$ & $5.23, \mathrm{dd}(17.5,1.1)$ & $111.8, \mathrm{t}$ & $\begin{array}{l}5.22, \mathrm{dd}(17.5, \\
1.1)\end{array}$ \\
\hline & & $5.12, \mathrm{dd}(10.9,1.1)$ & & $\begin{array}{l}5.05, \text { dd }(10.9, \\
1.1)\end{array}$ \\
\hline 13 & $25.9, \mathrm{q}$ & $1.68, \mathrm{~s}$ & $26.0, \mathrm{q}$ & $1.72, \mathrm{~s}$ \\
\hline 14 & $24.4, \mathrm{q}$ & $1.25, \mathrm{~s}$ & $28.2, \mathrm{q}$ & $1.29, \mathrm{~s}$ \\
\hline 15 & $67.4, \mathrm{t}$ & $\begin{array}{l}3.47, \mathrm{dd}(11.4,4.4) \\
3.42, \mathrm{dd}(11.4,6.2)\end{array}$ & 23.7, q & $1.29, \mathrm{~s}$ \\
\hline $\begin{array}{l}15- \\
\mathrm{OH}\end{array}$ & & $3.43, \mathrm{dd}(6.2,4.4)$ & & \\
\hline
\end{tabular}

\subsubsection{2,6-Dimethyl-7,10-epoxy-10-hydroxymethyldodeca-} 2,11-dien-6-ol (3)

Colorless oil, $[\alpha]_{\mathrm{D}}^{18}+5.9(c 0.03, \mathrm{MeOH})$. IR $(\mathrm{KBr}) v_{\max }$ $\mathrm{cm}^{-1}: 3445,2957,2922,2852,1634,1384,1099,1036 .{ }^{1} \mathrm{H}$ NMR (600 MHz,CDCl $)_{3}$ and $\left.{ }^{13} \mathrm{C} \mathrm{NMR} \mathrm{(150} \mathrm{MHz,} \mathrm{CDCl}_{3}\right)$ spectroscopic data, see Table 2. HREIMS $\mathrm{m} / z: 254.1873$ $[\mathrm{M}]^{+}$(calcd for $\mathrm{C}_{15} \mathrm{H}_{26} \mathrm{O}_{3}, 254.1882$ ).

\subsubsection{7,10-Epoxy-2,6,10-trimethyldodeca-2,11-diene-4,6- diol (4)}

Colorless oil, $[\alpha]_{\mathrm{D}}^{18}+0.4(c=0.01, \mathrm{MeOH})$. IR $(\mathrm{KBr}) v_{\max }$ $\mathrm{cm}^{-1}$ : 3432, 2957, 2923, 2853, 1630, 1463, 1384, 1111, 1038. ${ }^{1} \mathrm{H}$ NMR $\left(600 \mathrm{MHz}, \mathrm{CDCl}_{3}\right)$ and ${ }^{13} \mathrm{C} \mathrm{NMR}$ $\left(150 \mathrm{MHz}, \mathrm{CDCl}_{3}\right)$ spectroscopic data, see Table 2. 


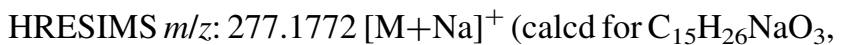
277.1780).

\subsection{Cytotoxicity}

The cytotoxicity assay was performed according to the MTT method in 96-well microplates. Five human cancer cell lines: human myeloid leukemia HL-60, hepatocellular carcinoma SMMC-7721, lung cancer A-549, breast cancer MCF-7 and human colon cancer SW480 cells were used in the cytotoxicity assay. All the cells were cultured in RPMI1640 or DMEM (Hyclone, Logan, UT, USA), supplemented with $10 \%$ fetal bovine serum (Hyclone) in $5 \%$ $\mathrm{CO}_{2}$ at $37^{\circ} \mathrm{C}$. Briefly, $100 \mu \mathrm{L}$ of adherent cells were seeded into each well of 96-well cell culture plates and allowed to adhere for $12 \mathrm{~h}$ before drug addition, while suspended cells were seeded just before drug addition with an initial density of $1 \times 10^{5}$ cells $/ \mathrm{mL}$. Each tumor cell line was exposed to the test compound at concentrations of $0.064,0.32,1.6,8$, and $40 \mu \mathrm{mol}$ in triplicates for $48 \mathrm{~h}$, with cisplatin (sigma, USA) as a positive control ( $\mathrm{IC}_{50}$ : SW480, $12.0 \mu \mathrm{mol}$; SMMC-7721, $10.2 \mu \mathrm{mol}$; HL-60, $3.1 \mu \mathrm{mol}$; MCF-7, $17.5 \mu \mathrm{mol}$; A-549, $9.1 \mu \mathrm{mol})$. After compound treatment, cell viability was detected and cell growth curve was graphed.

Acknowledgments This work was financially supported by National Natural Science Foundation of China (U1132607, 81102346), and Natural Science Fund of Yunnan province (2011FB099).

Conflict of interest The authors declare no conflicts of interest.

Open Access This article is distributed under the terms of the Creative Commons Attribution License which permits any use, distribution, and reproduction in any medium, provided the original author(s) and the source are credited.

\section{References}

1. Y.C. Dai, Chin. J. Appl. Ecol. 14, 1015-1018 (2003)

2. X. Wu, S. Lin, C. Zhu, Z. Yue, Y. Yu, F. Zhao, B. Liu, J. Dai, J. Shi, J. Nat. Prod. 73, 1294-1300 (2010)

3. A.R. Song, X.L. Sun, C. Kong, C. Zhao, D. Qin, F. Huang, S. Yang, Arch. Virol. 159, 753-760 (2014)

4. J. Wang, F. Hu, Y. Luo, H. Luo, N. Huang, F. Cheng, Z. Deng, W. Deng, K. Zou, Fitoterapia 95, 93-101 (2014)

5. J.J. Pei, Z.B. Wang, H.L. Ma, J.K. Yan, Carbohyd. Polym. 115, 472-477 (2015)

6. G. Aranda, I. Facon, J.Y. Lallemand, M. Leclaire, R. Azerad, M. Cortes, J. Lopez, H. Ramirez, Tetrahedron Lett. 33, 7845-7848 (1992)

7. H.E. Ramirez, M.M. Cortes, E. Agosin, J. Nat. Prod. 56, 762-764 (1993)

8. I. Markovic, Z. Djarmati, B. Abramovic, J. Serb. Chem. Soc. 73, 283-297 (2008)

9. K. Mori, M. Komatsu, Tetrahedron 43, 3409-3412 (1987)

10. D. Xu, S. Yu, Z.Y. Zhou, R. Liu, Y. Leng, J.K. Liu, Chem. Pharm. Bull. 57, 433-435 (2009)

11. R. Falk, A. Timm, S. Olov, Tetrahedron 58, 7785-7789 (2002)

12. A.A. Panasenko, E.C. Gorincioi, A.N. Aricu, E.A. Barcari, K. Deleanu, P.F. Lad, Russ. Chem. Bull. Int. Ed. 53, 2700-2705 (2004)

13. C. Manuel, D. Virginia, S. Claudio, A. Veronica, Nat. Prod. Commun. 6, 477-499 (2011)

14. Y.H. Kuo, C.H. Chen, S.C. Chien, Y.L. Lin, J. Nat. Prod. 65, 25-28 (2002)

15. Y.T. Lin, Y.S. Cheng, Y.H. Kuo, K.C. Lin, J. Chin. Chem. Soc. 21, 31-35 (1974)

16. J.P. Teresa, M.A.V.M. Alle, M.S. Gonzalez, I.S. Bellido, Tetrahedron 40, 2189-2195 (1984)

17. I.P. Tsypysheva, A.M. Kunakova, F.A. Valeev, G.A. Tolstikov, Chem. Nat. Compd. 37, 490-492 (2001)

18. D.S. Holmes, D.M. Ashworth, J.A. Robinson, Helv. Chim. Acta 73, 260-271 (1990) 